\title{
LOS ALCANCES DEL PLURALISMO LÓGICO
}

Omar Vásquez Dávila 


\section{LOS ALCANCES DEL PLURALISMO LÓGICO*}

Resumen: Según el pluralismo lógico no hay una lógica verdadera, sino varias. El pluralismo más conocido es el defendido por J.C. Beall y Greg Restall. Ellos son pluralistas respecto de la noción de consecuencia lógica. En este artículo, me adhiero al pluralismo lógico, pero sostengo que el pluralismo de Beall y Restall tiene problemas sin solución. Mostraré que hay importantes razones para buscar otro tipo de pluralismo.

Palabras clave: pluralismo lógico, consecuencia lógica, relativismo, monismo.

\section{THE SCOPES OF LOGICAL PLURALISM}

Abstract: According to logical pluralism there is not one true logic, there are many. The most well-known pluralism is the view defended by J.C. Beall and Greg Restall. They are pluralists about logical consequence. In this paper, I adhere to the logical pluralism but I hold that the Beall-Restall pluralism has many unsolved problems. I will show that there are important reasons to search for another kind of pluralism.

Keywords: Logical pluralism, logical consequence, relativism, monism.

Fecha de recepción: junio 30 de 2015

Fecha de aceptación: agosto 5 de 2015

Forma de citar: Vásquez, O. (2015). "Los alcances del pluralismo lógico". Revista Filosofía UIS. 14 (2). pp. 69-81.

Omar Vásquez Dávila: argentino. Estudiante de grado de la Universidad de Buenos Aires. Ha publicado en la Revista Filosofía UIS el artículo titulado "Términos vacuos: génesis y significado" (2009). Se desempeña como profesor de educación media. Sus principales áreas de interés son la filosofía de la lógica y la filosofía del lenguaje.

Correo electrónico: hildebrando2@hotmail.com 


\section{LOS ALCANCES DEL PLURALISMO LÓGICO}

\section{Introducción}

En este trabajo tomaré como supuesto el pluralismo en lógica ${ }^{1}$, es decir, la idea de que hay más de una lógica correcta, pero en un sentido amplio: las lógicas son correctas en tanto modelos que se aproximan a una caracterización precisa de algún fenómeno de interés para los lógicos (e.g. consecuencia en el lenguaje natural, consecuencia lógica deductiva, inductiva, analítica, etc.). Lo que interesa a los lógicos es que los sistemas, que estén bajo su consideración, tengan la capacidad de dar respuesta a los aspectos más importantes del fenómeno lógico en cuestión. Por ejemplo, si mi sistema lógico caracterizara la noción de consecuencia lógica, entonces, debería poder responder cuestiones acerca de la verdad, el significado, etc. Estas respuestas no tienen que ser acabadas, pues asumimos de antemano que hay un vacío entre el modelo y aquello que se pretende modelar.

Asumido el pluralismo, examinaré en qué debería consistir un pluralismo acerca de la lógica. Para esto tendré en cuenta el pluralismo lógico que muchos consideran como el más atractivo, a saber, el pluralismo de J.C. Beall y Greg Restall². La virtud de este pluralismo consiste en que es el intento mejor argumentado de un pluralismo en tanto respuesta al monismo. Sin embargo, este trabajo trata de poner en consideración la circunstancia en la que, si hemos asumido el pluralismo en lógica ${ }^{3}$, ¿es el pluralismo de Beall y Restall la mejor opción que podemos sostener?

\footnotetext{
${ }^{1}$ En la literatura acerca de estos temas, por lo general, se parte de una contraposición entre monismo y pluralismo. Véase Quine (1970), Cap. 6.

${ }^{2}$ El desarrollo completo de su postura aparece en J.C. Beall y Greg Restall (2006).

${ }^{3}$ Podemos dar por supuesto el pluralismo por razones intuitivas, teóricas o por la simple confirmación de la existencia de muchos sistemas lógicos. Alguien podría oponerse a esto diciendo que una postura pluralista está ineludiblemente ligada a su refutación del monismo. Sin embargo, las refutaciones al monismo consisten, por lo general, en argumentar en contra de la idea, según la cual, un sistema lógico en particular (e.g., el clásico) sea el único correcto. Ahora bien, este tipo de refutaciones no nos llevan necesariamente a sostener un
} 
La respuesta a esta pregunta será negativa por las siguientes razones: 1) Las ideas que Beall y Restall toman como punto de partida, y que por tanto funcionan como supuestos, son discutibles. 2) El pluralismo de Beall y Restall es un pluralismo dependiente de un relativismo; y los lógicos que dicen que hay más de un sistema lógico correcto desean estar bajo la etiqueta de pluralistas, no de relativistas. Finalmente, 3) Si partimos de los mismos supuestos y aceptamos su pluralismo, las lógicas que resultan correctas en su propuesta, son lógicas deudoras de las motivaciones teóricas y filosóficas de la lógica clásica (de esto no se sigue que si respetamos las motivaciones de las distintas lógicas, e.g., de la lógica relevante o intuicionista, seamos necesariamente monistas).

Por último, se esbozará un criterio (el cual es un proyecto a desarrollar en futuros trabajos) que nos proporcione una idea de cuál debe ser el alcance de un pluralismo en lógica, es decir, qué sistemas deben ser considerados lógicos y por qué.

\section{El pluralismo de Beall y Restall}

Tradicionalmente, la principal incumbencia de la lógica tiene que ver con el concepto de consecuencia lógica. Decir de qué da cuenta la consecuencia lógica consiste, normalmente, en especificar qué argumentos son válidos en un lenguaje dado. Según Beall y Restall, la naturaleza de la consecuencia lógica está capturada en el siguiente principio:

(V) Una conclusión $A$ se sigue de un conjunto de premisas $\Sigma$, si y sólo si cualquier caso en el que cada premisa de $\Sigma$ es verdadera, es también un caso en el que $A$ es verdadera.

Este principio sirve, a su vez, para introducir la noción de validez. Un argumento válido es uno cuya conclusión es verdadera en todo caso en el que todas sus premisas son verdaderas. Entonces, el rasgo característico de la validez es la ausencia de contraejemplos, casos en los que todas las premisas son verdaderas pero la conclusión falsa. Sin embargo, al aplicar esta definición nos encontramos con la dificultad de precisar cuáles (o de qué tipo) son los casos mencionados en dicho principio:

- Un sistema lógico debería especificar los casos mencionados en (V).

- Hay mínimamente dos especificaciones diferentes de tales casos.

pluralismo, pues podríamos pensar que otro sistema lógico es el único correcto; o estar a la espera del sistema lógico único y verdadero. 
La lógica resultante de dichas especificaciones podrían ser la clásica, la relevante o la intuicionista según se remplace "caso" por modelos tarskianos, situaciones o construcciones. Además, tanto los modelos como las construcciones pueden reducirse a situaciones si consideramos a éstas como completas y consistentes o como incompletas y consistentes, respectivamente ${ }^{4}$. Beall y Restall (2000) afirman que no todo reemplazo de caso en (V) cuenta como una lógica. Otras condiciones para pertenecer al ámbito de las lógicas son la normatividad, la formalidad y la necesidad. Según los autores, cualquier noción de validez que especifique un conjunto de casos y que cumpla con estos requisitos será un sistema de lógica legítimo y correcto.

Ahora bien, el principio (V) no da una caracterización completa del concepto de consecuencia lógica. Se necesita una precisa y sistemática explicación de qué argumentos son válidos. El pluralismo de Beall y Restall tiene en cuenta esta dificultad y se propone solucionarla. Para convencernos de optar por su pluralismo, Beall y Restall formulan el problema del siguiente modo:

a) La noción preteórica o intuitiva de consecuencia está dada por (V).

b) Una lógica es el resultado de una especificación de los casos que aparecen en (V). Tal especificación de casos puede ser vista como una manera de precificar (precisification) las condiciones de verdad de las oraciones del lenguaje en cuestión.

c) Hay, al menos, dos especificaciones diferentes de casos que pueden aparecer en (V).

Además de tener en cuenta a (V) se tiene que especificar qué es un caso y en qué consiste para una afirmación ser verdadera en un caso. Sin una respuesta a estas cuestiones no se podrá especificar qué es una lógica.

* $A \Lambda B$ es verdadero en $x$ si $y$ sólo si $A$ es verdadera en $x$ y $B$ es verdadera en $x$.

Donde $A$ y $B$ son afirmaciones y $x$ es un caso. Esta es una explicación de lo que significa ser verdadero en un caso que no sólo nos dice cómo funciona la conjunción, sino que también nos da una información sobre la validez. Podemos ir de $A$ y $B$ a $A$ : para cualquier caso $x$, si $A$ y $B$ es verdadero en $x$, entonces, $A$ es verdadero en $x$ (por la condición $\left.{ }^{*}\right)^{5}$. Hacer este tipo de especificaciones es, según Beall y Restall, hacer lógica. En este modo de formular el problema, la controversia acerca del pluralismo se agudiza con el punto (c). El que no acepte (c) -y además no crea que todas las lógicas son incorrectas- será considerado monista. Pero veamos cuáles son los supuestos en esta manera de presentar el problema.

\footnotetext{
${ }^{4}$ J.C. Beall y Greg Restall (1999).

${ }^{5}$ Lo mismo puede hacerse con las otras conectivas y la negación.
} 


\subsection{Los supuestos del pluralismo de Beall y Restall}

Con el supuesto (a) se privilegia la concepción modelo teórica o semántica de la consecuencia lógica sobre la explicación sintáctica o de prueba. Si Beall y Restall tratan de captar la noción intuitiva de la consecuencia lógica, deberían precisar por qué privilegian una concepción por sobre la otra y no justificar esta predilección apoyándose en el hecho de que los debates actuales se enmarcan en la tradición semanticista, como de hecho lo hacen.

Por otro lado, Beall y Restall sostienen que hay más de una relación de consecuencia en el lenguaje natural y cada lógica que resulta del reemplazo de caso en el principio (V) codifica una relación de consecuencia diferente. Sin embargo, los autores niegan que, del hecho de que distintas lógicas codifiquen distintas relaciones de consecuencia, se siga que esas lógicas no estén todas codificando la consecuencia lógica. Pareciera pues, que tenemos distintas lógicas, que codifican distintas relaciones de consecuencia, las cuales en conjunto, formarían una única relación de consecuencia (la cual es, precisamente, la noción que pretendemos explicar). La única forma de entender esto es que la consecuencia lógica es captada por aproximaciones, cada lógica aceptada por el pluralismo de Beall y Restall captaría distintos aspectos o rasgos de esta misma relación de consecuencia. Para ver el problema de este supuesto, analicemos la relación entre pluralismo y relativismo.

\subsubsection{Pluralismos dependientes de un relativismo ${ }^{6}$}

- Uno es relativista respecto de un fenómeno X si y solo si uno piensa que la correcta explicación de X está en función de distintos conjuntos de hechos Y. La correcta explicación de $\mathrm{X}$ sería relativa a $\mathrm{Y}$.

- Uno es pluralista sobre un fenómeno particular X si y solo si uno piensa que hay más de una correcta explicación de X. Monista si se piensa que hay sólo una.

- Uno es un pluralista no relativista si considera que hay más de una explicación correcta de $\mathrm{X}$ incluso si mantenemos todos los otros factores $\mathrm{Y}$ fijos.

- Pluralismo dependiente de un relativismo: una explicación de X es un pluralismo dependiente si involucra la existencia de muchas explicaciones correctas de $X$, pero esta multiplicidad es el resultado de un relativismo subyacente.

${ }^{6}$ Para esta caracterización de la noción de "dependencia" sigo a Cook (2010). 
Beall y Restall necesitan sostener que hay más de una relación de consecuencia en el lenguaje natural y cada lógica que resulta de (V) codifica un aspecto diferente de la misma relación de consecuencia general. Sostienen que no hay una característica esencial de la consecuencia lógica que codificar sino distintas e igualmente buenas aproximaciones a ella.

El pluralismo de Beall y Restall sería un pluralismo dependiente (como el de Carnap o el de Varzi) ${ }^{7}$. Pero, a diferencia de éstos, no es un pluralismo que se obtiene al variar el lenguaje en cuestión o al variar la división lógico/no lógico. Más bien, la noción de consecuencia lógica tiene cierta vaguedad o imprecisión que permite sostener diferentes especificaciones. Como resultado la lógica correcta es relativa a qué relación de consecuencia lógica del lenguaje natural uno está intentando codificar.

\subsection{Las lógicas deudoras de la lógica clásica}

Respecto de los conectivos lógicos, es sabido que los significados que los distintos sistemas lógicos atribuyen a éstos, no coinciden. Ni siquiera los tres sistemas que Beall y Restall priorizan para su pluralismo coinciden respecto del significado de los conectivos. En particular, el intuicionista no entiende la negación del mismo modo que el clásico y el relevante otorga al condicional un significado distinto al del condicional material de la lógica clásica. Sin embargo, al diferenciarse de un pluralismo de significados (en el cual cada lógica habla de distintas cosas), Beall y Restall necesitan de la mismidad de significados para que el significado de las fórmulas sea el mismo en distintas lógicas. Es en esta apelación a la mismidad del significado donde encontramos otro problema de su planteamiento y en el cual nos detendremos.

Beall y Restall suponen condiciones de verdad mínimas para conectivo. Por ejemplo, para la negación, su condición de verdad mínima sería: $\neg A$ es verdadera si y sólo si $A$ no es verdadera. Si relativizamos esto a modelos, construcciones o situaciones, tendremos la negación clásica, intuicionista y relevante, respectivamente. Aquí puede apreciarse un primer problema ¿Qué tiene que ver esta condición mínima con el significado, por ejemplo, de la negación intuicionista: para que $\neg A$ sea verdadera en una construcción $t$, $A$ no debe ser verdadera en ninguna construcción $v$ que extienda $t$ ? Como se ve no parece haber ninguna condición mínima que sea expresada por una definición respecto de la otra. Lo mismo sucedería si comparáramos el condicional intuicionista con el clásico.

${ }^{7}$ Véase Varzi, (2002) y Carnap, (1934). 
Las dificultades para establecer condiciones de verdad mínimas para los conectivos llevan a que Beall y Restall no se atrevan a formular condiciones mínimas para un conectivo tan importante para la lógica como el condicional. Ante esta dificultad podrían optar por alguno de los varios sistemas no clásicos que definen condiciones de verdad para el condicional. Existen teorías del condicional que no son veritativo-funcionales tales como el sistema C1 de Lewis y C2 de Stalnaker, los cuales tienen como base el sistema de esferas y se justifican a partir de la intención de evaluar el comportamiento del condicional en emisiones que prohíben situaciones inesperadas, es decir, se hace uso de cláusulas ceteris-paribus ${ }^{8}$. En términos de mundos posibles esta cláusula reconstruye la idea de similaridad entre mundos. Precisamente, esta idea de "similaridad entre mundos" explica la diferencia entre $\mathrm{C} 1$ y $\mathrm{C} 2$, sin embargo, en ambos sistemas no se cumple: $(p \rightarrow q$ : $(p \Lambda r) \rightarrow q),(p \rightarrow q \therefore \neg q \rightarrow \neg p)$ y tampoco la transitividad. Dado que no se cumplen estas inferencias, principalmente la transitividad, el principio (V) no podría asimilar a estos sistemas a su pluralismo. Por lo cual, en ningún sentido podríamos considerarlos sistemas lógicos, bajo el punto de vista de Beall y Restall.

Beall y Restall también sostienen que el significado de los conectivos en cada lógica rescata una parte del significado más abarcador que siempre es el mismo. El significado completo de un conectivo será el resultado de la unión de los significados que tenga en cada lógica. Sin embargo, dado que podemos reducir los modelos y construcciones a situaciones completas-consistentes e incompletas-consistentes, respectivamente, resultaría que la lógica relevante es la que en última instancia caracteriza el significado completo de los conectivos.

Ahora bien, dado que puede haber situaciones inconsistentes, tendría que asimilarse ciertas fórmulas que expresen contradicciones verdaderas. Entonces, tenemos aquí dos nuevos problemas. El primero, es que dicha forma de entender el significado completo de los conectivos deriva en un monismo relevante respecto del significado de los conectivos. El segundo problema es que tiene que aceptar contradicciones verdaderas, sin embargo, los sistemas lógicos que aceptan este tipo de contradicciones, como por ejemplo $\mathrm{LP}^{9}$, no están considerados en el tipo de pluralismo que proponen. ¿Se puede extender el principio (V) para abarcar lógicas paraconsistentes? La respuesta es No.

\subsection{Sistemas tergiversados por el pluralismo de Beall y Restall}

La lógica intuicionista surgió con la motivación de caracterizar la práctica matemática. Con esta mira, encontraron problemática la noción de verdad clásica con su componente extralingüístico. Pues, ¿cuál es la realidad extralingüística que corresponde a $2+3=5$ ? Así, rechazaron esa noción en su aplicación a las

\footnotetext{
${ }^{8}$ Véase Priest (2008), Capítulo 5.

${ }^{9}$ Véase Priest (2008), Capítulo 7.
} 
matemáticas. Entonces, propusieron que el significado de una oración no está determinado por las condiciones bajo las cuales es verdadera, sino por las condiciones bajo las cuales es probada, es decir, sus condiciones de prueba (Priest, 2008). Por ejemplo, una prueba de $\neg A$ es una prueba de que no hay una prueba de $A$. El condicional también tiene una formulación distinta. Una prueba de $A \rightarrow B$ es una construcción que, dada una prueba de $A$, puede ser aplicada para dar una prueba de $B^{10}$.

En la lógica intuicionista no se cumple el tercero excluido. Para probar esto debemos pensar en una semántica similar a S4, es decir, una semántica de las siguientes características: $\langle W, R, v\rangle$, aquí tenemos que $R$ es reflexiva y transitiva, y Rxy puede leerse como y extiende a $x$. $W$ es un conjunto de mundos $w, y$ cada uno de estos mundos puede entenderse como un estado de información o, generalmente, como una construcción. Que una fórmula valga en w significa que es probada en ese mundo. Para que el tercero excluido sea inválido debe haber un mundo $w$ donde falle, es decir, una valuación en ese mundo que a $p \vee \neg p$ le otorgue valor de verdad 0 . Esto implica que tanto la valuación de $p$ como de $\neg p$ tienen valor de verdad 0 . De esta manera, debe haber un mundo $w^{\prime}$ tal que $w R w^{\prime}$ y la valuación de $p$ en $w^{\prime}$ es igual a 1. Sea entonces $I$ una interpretación $<W ; R$; $v>$ donde $W=\left\{w, w^{\prime}\right\}, w R w, w^{\prime} R w^{\prime}$ y $w R w^{\prime}$, la valuación de $p$ en $w$ será igual a 0 y la valuación de $p$ en $w^{\prime}$ será igual a 1 . Del mismo modo, se puede encontrar un contra-modelo para la doble negación. De modo que, $\neg \neg A \therefore A$, fallaría en el sistema intuicionista.

Según Beall y Restall, un pluralista no debería aceptar que $A V \neg A$ no es verdadero, pues es consistente sostener, por un lado, que todas las verdades de la lógica clásica se sostienen y que todos los argumentos de la lógica clásica son válidos; y por otro, validar el uso de construcciones y con esto rechazar algunas inferencias clásicas. El punto crucial según Beall y Restall es fijar el contexto, es decir, sostener que las inferencias clásicas son válidas clásicamente, y no lo son, constructivamente.

Sin embargo, la motivación intuicionista fue rechazar la noción de inferencia clásica, buscando de este modo limitar el repertorio de pruebas. Para un clásico con pretensiones pluralistas es más fácil y cómodo decir que su sistema es más amplio y que la lógica intuicionista se restringe sólo a construcciones y que por tanto es una lógica válida en este sentido restringido. En cambio, un intuicionista con pretensiones pluralistas podría acusar a este pluralista clásico de ser un monista, pues al enfatizar en la semántica y en el criterio de preservación de verdad considera a su lógica más correcta que otras en tanto valida más inferencias que normativamente deben aceptarse si no fuera que a veces apelamos a

${ }_{10} \mathrm{El}$ condicional intuicionista valida las paradojas del condicional material, pero otras inferencias más problemáticas como $(A \wedge B) \rightarrow C \mathrm{~F}(A \rightarrow C) \mathrm{V}(B \rightarrow C)$ y $\neg(A \rightarrow B) \mathrm{F} A$, no se siguen. 
usos sintácticos restringidos. Usando un ejemplo, el pluralista clásico seguirá aceptando el tercero excluido, en cambio, un pluralista intuicionista no podría ser tolerante con este principio, ya que afecta la noción de prueba que fundamenta filosóficamente su sistema.

La principal motivación de la lógica relevante fue atacar la noción clásica de validez, criticar el criterio de preservación de verdad en modelos y reemplazarlo por uno de implicación relevante. Un relevantista no aceptaría el criterio de preservación de verdad, pues se comprometería con ciertas afirmaciones que su sistema prefiere evitar. En particular, un relevantista no acepta el Ex Falso (de una contradicción se sigue cualquier afirmación) ni el silogismo disyuntivo.

El pluralismo de Beall y Restall parece sostener que la lógica relevante no es rival de la clásica, pues podría aceptar la validez del Ex Falso bajo situaciones consistentes y completas, es decir, bajo la semántica de los mundos posibles. Ahora bien, en la formulación del principio (V) prevalece el criterio de preservación de verdad, el cual se diferencia del criterio de validez motivador de la lógica relevante. Respecto de esta estrategia cabe decir dos cosas. Primero, que la prevalencia del criterio de preservación de verdad deja a las lógicas no clásicas como meras alternativas a la clásica, y no como rivales, que es como ellas se pretenden.

En segundo lugar, proponer un pluralismo que acepte tres lógicas sólo porque su semántica puede constar de modelos, construcciones o situaciones no es un buen argumento contra el monismo. Para ver esto, pensemos en un monista relevante como Mortensen (1983). Este autor sostiene que la lógica relevante es la lógica correcta pues habla de todas las situaciones posibles. La lógica relevante, de este modo, describe las valideces universales correctas, mientras que la lógica clásica es un caso especial que se sostiene sólo sobre un dominio restringido, es decir, trata de formas de argumentos que, en circunstancias más restrictas, nos llevan de verdad a verdad.

Este ejemplo muestra en qué sentido restringir las aplicaciones de una lógica no nos hace necesariamente pluralistas. Mortensen acepta que podemos hacer uso del silogismo disyuntivo con disyunción extensional bajo situaciones normales, pero sigue siendo un monista relevante. Por lo mismo, Beall y Restall no hacen más que explicitar su preferencia por la lógica clásica, al aplicar restricciones a las situaciones para generar distintos sistemas. 


\section{Conclusión y esbozo de un pluralismo más abarcante que el de Beall y Restall}

Las principales críticas de este trabajo han sido: señalar algunos supuestos fáciles de cuestionar en la propuesta de Beall y Restall. Además, mostrar que la apelación a las condiciones mínimas de significados para los conectivos es infundada, debido a los distintos significados que distintas lógicas atribuyen a sus términos lógicos. También, cuestionar la idea, según la cual, los conectivos de distintas lógicas captan distintas caras de un mismo significado.

Además, argumentar que el principio (V) y las condiciones para considerar a una lógica correcta, son infieles con las motivaciones filosóficas de otros sistemas, y dejan fuera sistemas lógicos que sería importante considerar dados los distintos fenómenos que se generan alrededor de nociones tales como la de validez, verdad, etc. Esta crítica se basó, principalmente, en la idea de que Beall y Restall muestran cierta preferencia hacia la lógica clásica, la cual se basa en la adopción del criterio de preservación de verdad y la relativización de la semántica a la de situaciones completas y consistentes. Esto les permite considerar a los sistemas intuicionista y relevante como extensiones del sistema clásico generados a partir de ciertas restricciones en la semántica.

En contra de esto se argumenta que es arbitrario imponer el criterio de preservación de verdad, pues es infiel a las motivaciones de los otros sistemas. Dado que el requisito de necesidad está íntimamente ligado a este criterio, se puede llegar a la conclusión de que lo único que queda en pie de la propuesta de Beall y Restall es que, efectivamente, la lógica se encarga de qué se sigue de qué, o sea de la validez, y que los sistemas que den cuenta de este fenómeno tienen que cumplir a condiciones de normatividad y formalidad.

En este sentido, se observa que hay sistemas tales como LP y el sistema de las lógicas condicionales que cumplen con estos requisitos, es decir, dichos sistemas teorizan la noción de validez, son normativos y formales. Por otro lado, hay sistemas que no se caracterizan principalmente por su apelación a la semántica, por ejemplo, la lógica relevante. Según Read (2006), la semántica relevante es sólo un instrumento y no es necesario proporcionar una interpretación coherente y razonable de ella. Por lo tanto, debe considerarse, no como una lógica de las situaciones inconsistentes, sino como la lógica de la inferencia relevante. De todo esto se sigue que el pluralismo de Beall y Restall no es la mejor opción, en cuanto a pluralismos lógicos se refiere, por la cual podamos optar, si tenemos realmente intuiciones pluralistas, es decir, si se considera que hay más de un sistema (o muchos) que merecen ser llamados sistemas lógicos. 
Ahora bien, ¿cómo armonizar todos estos sistemas en un pluralismo lógico? Al respecto, se debe renunciar a la pretensión de explicar acabadamente una noción, en este caso, la noción de consecuencia lógica. Se advierte que hay muchos problemas sin resolver cuando se pretende (como Beall y Restall) sostener que la relación de consecuencia se logra explicar a través de distintas aproximaciones. Tal vez, dado que hay distintas relaciones de consecuencia en el lenguaje natural y distintas lógicas que caracterizan estas distintas relaciones de consecuencia, para cada relación de consecuencia debe haber más de una lógica que la caracteriza adecuadamente. Y dado que hay un vacío entre el modelo y lo que se modela, la corrección de una lógica tiene que ver con el rango de conceptos interesantes que están relacionados con la consecuencia y que pueden clarificarse a partir de la lógica que esté siendo usada. Así se obtiene un pluralismo dependiente de los fines teóricos.

Podría decirse que cada relación de consecuencia viene unida a un lenguaje y que es la teorización hecha a través de otro tipo de lenguaje la que origina ese vacío y la posibilidad de distintas lógicas igualmente correctas. Si bien con esto se cae en un pluralismo de contextos, se agrega que los sistemas lógicos deben tratar la noción de validez y los conceptos relacionados a esta noción. La pregunta que surge es: ¿̇y cuál de estas explicaciones del concepto de validez será la mejor?

Ante esto podemos responder lo mismo que Cook (2010) respecto de los modelos. Según este autor, puede haber modelos múltiples e incompatibles entre sí que compiten para caracterizar el mismo fenómeno. Esto se debe a que siempre habrá una brecha infranqueable entre el modelo y lo que se modela. Así, cuando se modela un fenómeno en particular, se puede llegar a modelos distintos e incompatibles debido a la adopción de distintos objetivos o distintas simplificaciones. El hecho de realizar distintas idealizaciones también generaría la incompatibilidad. De este modo, dado que hay distintos modos igualmente válidos de abordar un fenómeno, y dados los distintos fenómenos asociados con el concepto de validez, no sería raro un escenario con más de una lógica correcta o un escenario donde no haya una lógica que sea mejor a otras.

Esta consideración permite integrar al pluralismo otros sistemas, como por ejemplo LP, e incluso una lógica relevante que consta de una relación de accesibilidad ternaria. Si bien este tipo de relación ternaria es fuertemente cuestionada, se justifica su uso debido al éxito explicativo que tiene para la teoría expuesta, dejando de lado cuestiones metafísicas que justifiquen su existencia, y apelando simplemente a consideraciones pragmáticas. Esto no significa que no tengamos una justificación filosófica o metafísica, sino solamente que su uso, hasta que este tipo de explicaciones aparezcan o se generen, está garantizado por el éxito en la explicación $\Phi$ 


\section{REFERENCIAS}

Beall, J.C. y Restall, G. (1999). "Defending Logical Pluralism", en Brown, B. y J. Woods (eds.) Logical consequence: rival approaches, Stanmore: Hermes, 2001, pp. 1-22.

Beall, J.C. y Restall, G. (2000). "Logical Pluralism". Australasian Journal of Philosophy. 78. pp. 475-493.

Beall, J.C. y Restall, G. (2006). Logical Pluralism. Oxford: Oxford University Press.

Cook, R. (2010). "Let a Thousand Flowers Bloom". Philosophy Compass. 5/6. pp. 492-504.

Mortensen, C. (1983). "The validity of disyunctive syllogism is not so easily proved". Notre Dame Journal of Formal Logic. 24. pp. 35-40.

Priest, G. (2008). Introduction to non-classical logic: from if to is. Oxford University Press.

Quine, W.V.O. (1970). "Deviant logics". En Philosophy of Logic. New Jersey: Prentice Hall, cap. 6).

Read, S. (2006). "Monism: The One True Logic". En DeVidi, D. and T. Kenyon (eds.). A Logical Approach to Philosophy: Essays in Honour of Graham Solomon, Springer 2006, 193-209.

Varzi, A. (2002). "On Logical Relativity". Philosophical Issues. 10. pp. 197-219. 